\title{
sciendo
}

\section{Interacting Constraints and Relative Age Effect in Elite Cross-Country Skiers and Freeskiers}

\author{
by \\ Truls Roaas ${ }^{1}$, Håvard Lorås ${ }^{2}$, Tore Kristian Aune ${ }^{1}$, Boye Flakken ${ }^{1}$, Terje Dalen ${ }^{1}$
}

\begin{abstract}
Different characteristics of sports disciplines potentially lead to skewness in birth month distribution (relative age effect, RAE). These characteristics can be considered from a constraint-based approach with interacting environmental, task, and individual constraints as a theoretical framework with which to examine variations in RAE. The main aim of the present study was to examine the theoretical predictions of the constraint-based framework by investigating the birth month distribution in cross-country skiers and freeskiers at elite junior and senior levels. The sample was comprised of top ranked Norwegian U15-U19/20 cross-country skiers and junior-level freeskiers. Birth months of top ranked international senior-level skiers in cross-country and freeskiing were also collected. Results indicated an over-representation of skiers born in the first half versus the second half of the year at all junior ages in cross-country skiing. There was no significant difference in the distribution of birth months in freeskiing or in senior cross-country skiers or freeskiers. Based upon the interacting constraint framework, the skewness towards more earlyborn athletes in junior cross-country skiing could be due to strict age-grouped and results-oriented developmental programs beginning at an early age (environmental constraints) as well as high-demand for physical capabilities (task constraints) that favour more physically mature athletes (individual constraints). For freeskiing, the interacting environmental (less structured and more individualistic-oriented development), task (high technical/motor skill demands), and individual (no advantage of advanced physical maturation) constraints potentially operate in the opposite direction compared to cross-country skiing.
\end{abstract}

Key words: RAE, developmental variability, performance, contextual specificity.

\section{Introduction}

All sports have different features. Some differences are obvious, such as performance execution or the environmental characteristics where sports are executed. However, it is not easy to identify how these differences influence on the demand of particular athletes' qualities or how the contextual characteristics impact athletes' opportunities. In order to reduce developmental differences between athletes during childhood and youth, annual age-divided cohorts are used. Despite this organisation, research has shown skewed birthdate distributions within age cohorts (Cobley et al., 2009). The skewness is typically manifested as a substantial proportion of athletes being born early according to the annual age-grouping cut-off date, and such disproportional distributions are termed the 'relative age effect' (RAE). Since its first inception in the mid-1980s (Barnsley et al., 1985), this effect has been demonstrated in abundance across a variety of individual and team sports (Cobley et al., 2009; Delorme et al., 2010; Wattie et al., 2015).

There appears to be no consensus in terms of scientific approaches as to what can be considered effective strategies that counteract the potential negative effects of being born late in

1 - Department of Sport Sciences, Sport and Human Movement Science Research Group (SaHMS), Faculty of Education and Arts, Nord University, Levanger, Norway.

2 - Department of Teacher Education, Faculty of Social and Educational Sciences, Norwegian University of Science and Technology, Trondheim, Norway. 
one's cohort (Webdale et al., 2020). One reason for this is perhaps that RAE studies, in general, have been described as 'atheoretical', which potentially leaves a gap in both the understanding of components which impact upon relative age within and across sports and how relative age influences performance-related outcomes in sports. Thus, it seems clear that a comprehensive interdisciplinary theoretical platform is necessary to understand both the RAE phenomenon and its consequences in order to develop programs which may counter the RAE in specific sports. In this regard, Wattie et al. (2015) proposed a constraint-based model with reference to tenets from Newell (1986) as an approach to relative age research. The term 'constraints' captures conditions or factors related to the opportunities or limitations in a given context. Changes in any of these constraints are considered to alternate the relationship between various interacting constraints and, thus, possibly change the outcome. From this perspective, bidirectional interactions between individual, environmental, and task constraints influence the presence and the degree of the relative age effect in sports.

Athletes' individual constraints include structural factors (i.e., height, weight, timing/tempo of maturation) and functional factors (i.e., psychological factors) (Newell, 1986). In some sports contexts where height, body mass, strength, and power are performance advantages (e.g., volleyball, basketball, track and field, alpine skiing), early maturing athletes at any given age are likely to have a biological advantage over those athletes who mature later (Baxter-Jones, 1994). Athletes born early in a selection year will be, on average, more physically developed compared with athletes born later in the same cohort (Dalen et al., 2017). Individual physical maturation thus influences RAE in sports through what has been termed the 'maturation-selection hypothesis', which specifically states that organisational bodies responsible for selecting young athletes (e.g., coaches, talent scouts, and sport officials) are far more likely to select athletes who are more physically advanced compared with athletes in the same annual cohort. Athletes' variability in physical growth and maturation have bi-directional interactions with their ability to perform within the task constraints, influencing the presence and magnitude of RAE (Wattie et al., 2015). Task constraints are composed of the specific nature of a particular sport, performance characteristics (e.g., whether performance requires specific qualities associated with physical, technical or motor skills), as well as rules and regulations of the sport. It is hypothesised that in traditional sports where increased physical capabilities correlate positively with selection and performance, RAEs are more likely to flourish (Wattie et al., 2015). Indeed, research has identified RAE in a number of physically demanding sports such as basketball (Delorme and Raspaud, 2009), tennis (Dudink, 1994; Edgar and O'Donoghue, 2005), alpine skiing (Müller et al., 2015), ice hockey (Nykodým et al., 2020) and handball (Schorer et al., 2009). As shown by Fonseca et al. (2019), the prediction is that high physical demands in a specific sport (task constraints) interact with maturation-selection mechanisms (individual constraints), leading to RAEs in sports that match these characteristics.

Environmental components associated with broader social constructs can affect an athlete's development, including physical training and the competition environment, socio-cultural environment, policies, and the influence of significant others on athletes' lives. Environmental constraints also include factors such as the cultural significance of a sport in a country or region as well as different developmental and talent selection systems of a sport. In particular, the policies which regulate youth sports participation, athletes' selection, and developmental programs are hypothesised to influence RAE. For example, in a high-speed collision sport such as American football, with tremendous demands for physical capabilities in achieving high performance, one would expect the presence of RAE given the above-mentioned considerations. However, the absence of RAE in this sport has been attributed to the organisational system in American youth football in which players are placed into annual age cohorts and weight categories until late adolescence, when maturation variability typically levels out (Jones et al., 2019; MacDonald et al., 2009; Steingröver et al., 2016). Research and empirical evidence on how changes in environmental constraints interact with task and individual constraints appear to be limited (de la Rubia et al., 2020). 
Cross-country skiing and freeskiing are sports with considerable differences in terms of varying demands for physical/technical/motor capabilities and the physical environment of training and competition. Thus, these two sport contexts are particularly suited to investigate and compare RAEs because of their contextual differences in task execution (performance characteristics) and the environment (milieu, rules, regulations, etc.). Former studies within the cross-country skiing context have shown RAE in senior athletes (Baker et al., 2014) and in youth athletes (Raschner et al., 2012; Romann et al., 2018) when grouped together with other skiing disciplines. The RAE tendencies in cross-country skiing could be seen as relatively stable, although the data in this sports discipline are scarce, and to our knowledge, no studies have investigated RAE in elite cross country skiing across separate age groups and skill levels. This latter point has been shown to influence the variation in relative age effect in other sports (de la Rubia et al., 2020; Kearney et al., 2018). In addition, the current literature appears to contain no investigations of potential RAE in freeskiing.

Cross-country skiing, the most traditional and popular skiing discipline in Norway, can be categorised as a highly organised sport where coach-led training procedures are commonly applied from a relatively early age (5-6 years old) in local clubs situated across the country. Young cross-country skiers normally follow a traditional and specified training regime influenced by a prescriptive 'best practice' with high coach involvement. Similarly, competitions, scoreboards, and assessment procedures are carried out at a relatively early age. The regional talent selection procedures begin around the age of 11-12 years and are soon followed by selection for youth national championships. By contrast, freeskiing is a modern skidiscipline developed by adventure skiers worldwide that explores off-piste terrain for jumping stunts, and it typically signals a specific personal identity (Thorpe and Wheaton, 2011; Wheaton, 2004). Freeskiing could be defined as a still relatively self-organised sport, with little coach-led practice or organised athletes' developmental programs initiated from an early age. Athletes' performance development in freeskiing largely consists of, and depends upon, practicing tricks, jumps, and other features to create challenges to overcome. In Norway, freeskiing national competitive age categories are junior (13 to 17 years) and senior (18 years and up). Freeskiing is thus an individualised sport which requires a high level of technical motor skills to complete tricks, jumps, and other movements. Competitions occur in a slope consisting of different obstacles, and performance in competition is scored on criteria such as progression, amplitude, variety, execution, and difficulty (afpjudges.com). Conversely, in crosscountry skiing (although also primarily an individualised sport), the athlete with the highest average speed wins the competition, and competitive events typically last from $\sim 3 \mathrm{~min}$ (sprint) to $\sim 2 \mathrm{~h}(50 \mathrm{~km})$; it is, thus, mainly an endurance-related activity with fixed ski tracks in variable snow conditions and terrain (approximately one-third uphill, flat, and downhill). High performance in cross-country skiing is primarily related to endurance capacity and high peak oxygen uptake (VO2PEAK), movement-specific strength, and only a few repetitive skiing techniques (Sandbakk and Holmberg, 2017; Solli et al., 2018).

Based on the presented considerations, which highlight the role of a constraint-based approach with interacting environmental, task, and individual constraints as a theoretical framework with which to explore variations in relative age effect (Wattie et al., 2015), the current study set out to examine the constraint-based frameworks' theoretical predictions by investigating the birth month distribution in cross-country skiers and freeskiers at elite junior and senior levels. Specifically, it was hypothesised that elite cross-country skiers would demonstrate systematic skewness towards earlier birth months due to a high degree of performance-oriented developmental programs from an early age (environmental constraints) and high demand for physical capabilities (task constraints) which interact to result in more physically mature athletes (individual constraints) being potentially selected (maturation-selection hypothesis). In contrast, freeskiing is considered to have a less organised practice environment and more dynamic developmental programs (environmental constraints). Freeskiing has high technical/motor skill demands with 
individualised movement patterns (task constraints) that in principle result in no advantage of advanced physical maturation (individual constraints), therefore, it was hypothesised to be no systemic skewness in birth month distribution.

\section{Methods}

The overall sample was comprised of birthdates of Norwegian male elite cross-country and freeskiers. In order to be defined as an elite skier in the present study, the athlete had to be ranked among the 50 best in his cohort. In total, data of 376 male skiers were distributed across the two skiing disciplines and different age groups (junior, $\mathrm{n}=276$; senior, $\mathrm{n}=100$ ). Birthdates of top ranked national cross-country skiers were collected in the Norwegian U15-U19/20 age categories, as well as birthdates of the Norwegian top ranked junior-level freeskiers. Junior skiers were athletes participating in either the unofficial Norwegian championship or in the Norwegian Cup. Birthdates of junior athletes were collected from rankings based on the result lists collected from the Norwegian Ski Federation (www.skiforbundet.no). The international senior skiers in cross-country and freeskiing were athletes participating in international competitions (World Cup, WC), and their birthdates were collected from the official website of the International Ski Federation (www.fisski.com). To examine RAE, athletes' birthdates were categorised according to the cut-off date of the $1^{\text {st }}$ of January, which is the international cutoff date for both Norwegian and international skiing. Given the study focus on elite athletes, the resultant sample size was relatively moderate. The month of birth was therefore extracted and categorised into first and second half-years according to the cut-off date. The study was conducted according to the Helsinki Declaration and approved by the Norwegian Social Science Data Services (NSD, reference number 519335).

\section{Statistical analysis}

To examine relative age effects, the distribution of the participants' birth month across the first and second half-year was examined by chi-square tests $(\chi 2)$ against an even distribution, with Cramer's $V(\phi)$ as a measure of effect size interpreted according to Cohen (1978) as small $<0.05$, medium $\geq 0.06$ to $<0.24$, and large $\geq 0.25$. To examine the hypothesis that elite crosscountry skiers would demonstrate systematic skewness towards earlier birth months, chi-square tests were applied separately to the U15, U16, U17, U18, U19/20 and senior cross-country subsamples. Similarly, in order to examine the hypothesis that there is no systemic skewness in birth month distribution in freeskiers, junior and senior subsamples were subjected to chi-square analysis. Predictive Analytics Software (IBM SPSS Statistics for Windows, Version 25.0. Armonk, NY: IBM Corp.) was used for all statistical procedures with $p<0.05$ as a statistical significance criterion.

\section{Results}

In cross-country skiing, significantly more skiers [152 vs. $81, \chi^{2}=21.6, p<0.05, \phi=0.30(95 \%$ CI: $0.21,0.42)]$ were born in the first half versus the second half of the year across all junior ages (Figure 1A). Specifically, this difference was significant for U15 [31 vs. 17, $\chi^{2}=4.1, p<0.05, \phi=$ 0.29 (95\% CI: $0.25,0.53)$ ] and U17 [35 vs. 11, $\chi^{2}=$ $12.5, p<0.05, \phi=0.52$ (95\% CI: 0.33, 0.77)], albeit non-significant for U16 [31 vs. 19, $\chi^{2}=2.9, p>0.05$, $\phi=0.24$ (95\% CI: $-0.24,0.48)$ ], U18 [26 vs. $16, \chi^{2}=$ 2.4, $p>0.05, \phi=0.24$ (95\% CI: $-0.24,0.49)$ ], and $\mathrm{U} 19 / 20\left[29\right.$ vs. $18, \chi^{2}=2.6, p>.05, \phi=0.23$ (95\% CI: $-0.23,0.47)$ ] (Figure 2). In comparison, results indicated no statistical differences in the distribution (first half vs. second half) of the junior freeskiing sub-sample [19 vs. $24, \chi^{2}=0.6, p$ $>0.05, \phi=0.12$ (95\% CI: -0.26, 0.33)] (Figure 1A). At the senior level, no significant differences were identified in distribution across the first and second halves of the year in either the crosscountry [23 vs. $27, \chi^{2}=0.3, p>0.05, \phi=0.08(95 \%$ CI: $-0.24,0.25)]$ or the freeskiing [27 vs. $23, \chi^{2}=0.3$, $p>0.05, \phi=0.08$ (95\% CI: $-0.24,0.25)]$ sub-samples (Figure 1B). 

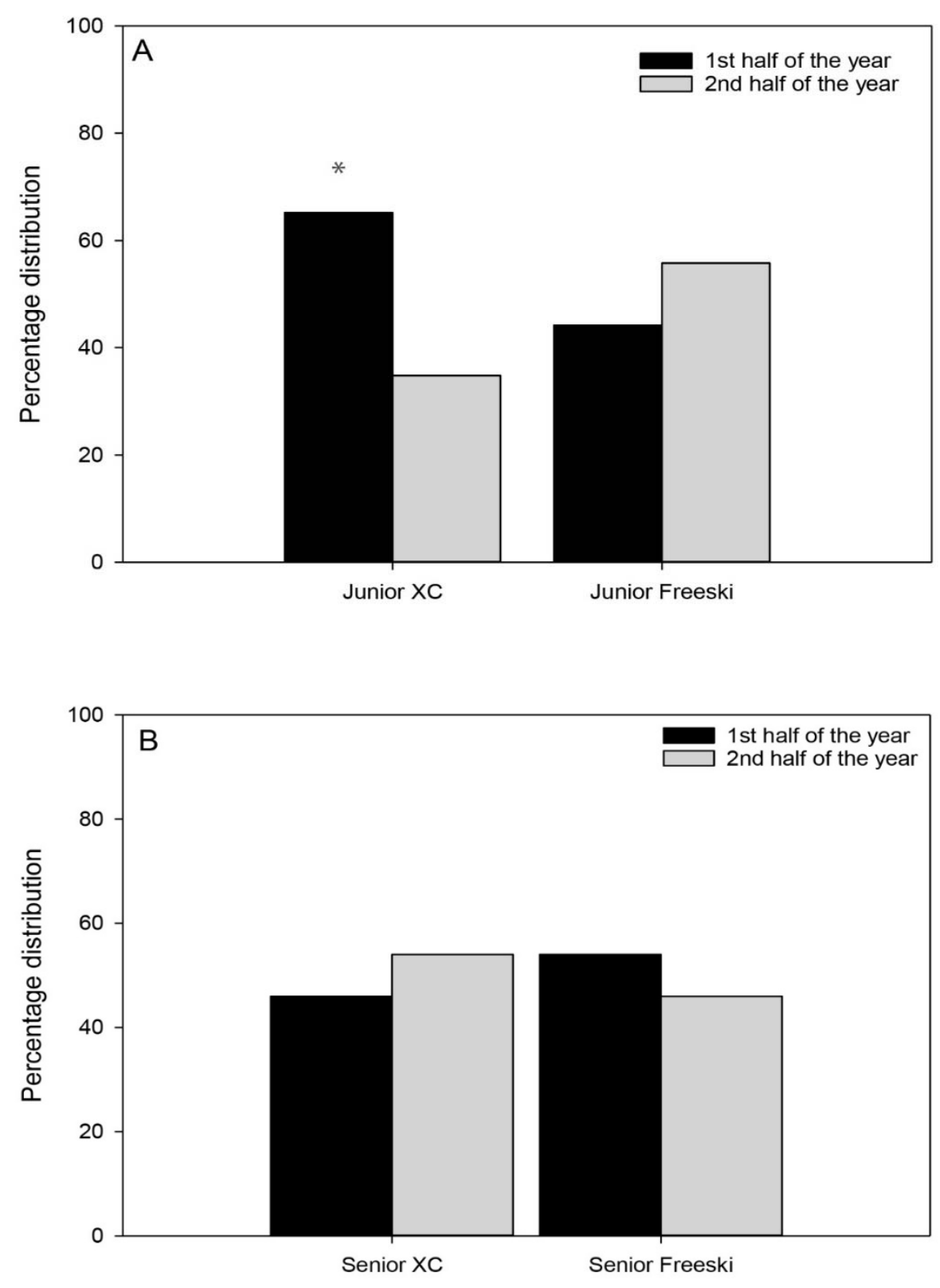

Figure 1

Percentage half-year distribution of top 50 best junior skiers in cross country and freeskiing (Figure 1A), and distribution of top 50 international skiers in cross country and freeskiing (Figure 1B). * indicates significantly higher than the second half-year. 


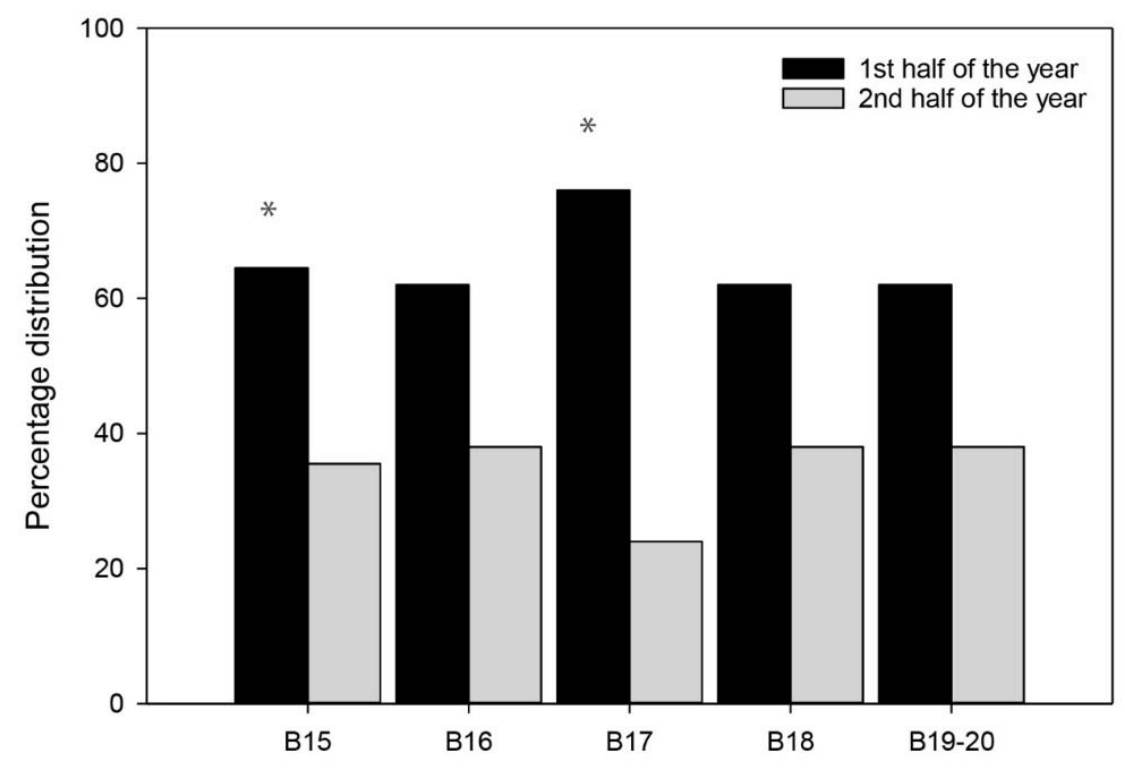

Figure 2.

Percentage of half-year distribution of cross-country junior skiers in different age groups.

* indicates significantly higher than the second half-year. $B=$ Boys.

\section{Discussion}

The aim of the present study was to examine the theoretical predictions of the constraint-based framework by investigating the birth month distribution in Norwegian elite crosscountry skiers and freeskiers at junior and senior levels. As hypothesised, in elite cross-country skiers at the junior level, there was systematic skewness and over-representation of athletes born in the first versus the second half of their birth year, while for freeskiers, there were no differences in the same distribution. At the senior level, no differences in the distribution of birthdates across the first and second halves of the year were identified in either cross-country skiers or freeskiers. These results are in agreement with predictions of the constraint-based model (Wattie et al., 2015), where bi-directional interactions between the respective constraints (environmental, individual, and task constraints) probably affect athletes' opportunities for development and result in competitive differences for cross-country skiers compared to freeskiers at a young age.

On average, early-born athletes are more physically developed in their age categories, and in endurance sports like cross-country skiing, physical development might be an important predictor of performance at the early stages of athletes' careers (e.g., Raschner et al., 2012). For cross-country skiing, possible consequences could be the promotion of a selection bias that might confuse 'maturation' with 'talent' (Dalen et al., 2017; Hirose, 2009). This could be problematic if individualised skiing sports which depend highly on physical performance demands, with peak performance later in development, organise 
themselves in a way that increases RAEs. Apparently, task constraints (e.g., physical demands) associated with freeskiing are not influenced by the RAE.

Freeskiing is classified as a compositional technical skill sport with task and individual constraints similar to other sports like gymnastics, figure skating and snowboarding. Several studies in gymnastics have demonstrated that it is advantageous to be of a smaller stature. It has been documented that RAE seemingly works in the opposite direction, and relatively late-born athletes are in the majority (Baker et al., 2014; Hancock et al., 2015). The present study does not report anthropometric data directly, but assumes that late-born athletes at the junior level are of a smaller stature. The present study showed an advantage for junior cross-country skiers born early in their birth year, presumably because of their earlier maturation compared to the competing peers born late in the same cohort. On the other hand, the results showed a tendency for an inverted RAE for junior freeskiers. Hence, individual constraints (e.g., anthropometrics) potentially play a significant role, and there is no support in other studies investigating RAEs in freeskiing. Further empirical research with anthropometric measurements and other complementary data is necessary to uncover how differences in the task and individual constraints impact the RAE in sports with different skill demands.

The findings of RAEs in junior crosscountry skiing suggest that an organisational constraint such as a strict age categorisation is associated with the higher degree of RAE seen in elite youth cross-country skiers. With traditional rule-bound constraints, together with an increase in the competition level, a stronger RAE is likely (Baker et al., 2014; Helsen et al., 1998; Musch and Grondin, 2001). Based on previous research (Baker et al., 2014), significant RAEs were found in senior male athletes in cross-country skiing; one resulting suggestion is that age categorisation is an environmental constraint leading to a prolonged RAE in top-level senior cross-country skiing. However, the results of the present study found no significant RAE in top-ranked worldclass senior cross-country skiing. Presumably, there could be several explanations for the diminishing RAE in elite senior athletes. The most obvious explanation relates to the organismic constraints based on the fact that RAE between early- and late-born athletes decreases with age and that senior-level athletes have fully matured. Secondly, other environmental constraints could cause intra-modalities between junior and senior levels, like the 'underdog effect' (Bjerke et al., 2017; McCarthy and Collins, 2014) and 'late bloomers' (Gulbin et al., 2013).

Given that the age grouping for competition in freeskiing is not restricted within annual age groups, but in junior grouping (13-17 years), there would seemingly be little advantage to being born early in the year. However, one could expect a clustering of freeskiers born in the latest years of the junior age group (i.e., 16-17 years), but this does not seem to be the case based on the distribution of birth months in this study sample. The birthdate distribution in freeskiing seems to be evenly spread out between the different year-groups and into the senior elite. The contrast between age grouping policies in crosscountry skiing and freeskiing would support the importance of bi-directional interaction between environmental and individual constraints.

The two different ski disciplines investigated in this study are defined as more traditional (cross-country skiing) or modern (freeskiing) sports. Thus, regarding differing sports cultures, modern skiing has environmental and task constraints similar to other action sports in the present time. These cultures are associated with factors such as self-organised 'do-it-yourself' philosophies, social media exposure and subcultural differences from traditional rulebound sport cultures (Thorpe and Wheaton, 2011). Freeskiing athletes have, from the beginning, created the contextual characteristics of the sport, manipulating task constraints (i.e., tricks, jumps and other features in the milieu) to invent challenges and drive themselves to their limits. Moreover, the need for strict organisation, coaching instructions and assessment is redundant. However, with an increased organisational influence in freeskiing competitions (i.e., from FIS), athletes find themselves at a crossroad. The seemingly absent RAE in freeskiing will perhaps be challenged in the future if the characteristics of the sport are changed. This proposition is related to the historical changes that have taken place in cross- 
country skiing, which became a more rule-bound sport in the last century when governing federations made continuous competition changes. Webdale et al. (2020) proclaim that, in general, environmental constraints do not change the nature of a task.

Organisational changes (environmental constraints) could influence task constraints, as seen in the contexts of cross-country versus freeskiing. This is exemplified through governing restrictions in techniques, cut-off dates, changes in the competition rules, and administrative decisions on what is most appropriate. Consequently, selection pressure increases in cross-country skiing from a young age, and the search for a few 'appropriate' cross-country athletes enhances several documented RAE effects like the 'Pygmalion effect' and the 'Matthew effect' (Hancock et al., 2013). The 'Pygmalion effect' indicates self-fulfilling prophecies based on higher expectations placed upon an individual that lead to better attainment by the individual (Rosenthal and Jacobson, 1968). The 'Matthew effect' refers to differences in achievements as a function of unevenly distributed facilities in sports, most notably better coaches, better training facilities, better opponents, and the like (Merton, 1968).

The results from the current study need to be interpreted against a relatively modest sample size. However, the total $n=376$ emerged as a consequence of examining the predictions of the constraint-based model (Wattie et al., 2015) in two sports (freeskiing and cross-country skiing) that are situated in a Norwegian context and still dissimilar in task and environmental characteristics. Furthermore, the study was restricted to top-ranked elite level performers, as the skill level has been found to impact RAE (de la Rubia et al., 2020). These sample characteristics are necessary in order to be able to examine the possible interactions of task, environmental and individual constraints and their effect on RAE. As discussed by Gibbs et al. (2015), RAE data have some unique features in sports research, as samples are not typically drawn from a larger population, but represent the entire population studied. In some cases, this might even render the use of inferential statistics unnecessary.

In the context of the current study, most of the Norwegian elite junior/senior athletes in freeskiing and cross-country skiing were included, and thus a substantial proportion of this specific population. Still, it is certainly necessary to examine the predictions from the constraintbased model in both larger and more international samples of elite athletes, and the current study represents an initial step in this direction. Future studies should provide nuanced and longitudinal data on interacting constraints which affect RAE. However, adding to the current literature on relative age effect, this study examined a novel context based on a constraint-based theoretical framework.

It was hypothesised that systematic skewness would exist towards earlier birth months in elite junior cross-country athletes in each annual cohort. In the junior cross-country skiing sample, $65 \%$ of the athletes were born in the first half of the year. This indicates that the sport-specific criteria for performance favour the early-born athlete in junior levels in cross-country skiing. However, there was no skewness in birth distribution among either senior cross-country skiers or junior and senior freeskiers. The respective constraints potentially operate differently, but it is difficult to identify the arbitrative constraint causing the differences in RAE within the present dataset and the current constraint-based theoretical framework. According to our findings, it could be beneficial for coaches and policymakers in cross-country skiing to carry out long term monitoring of performance characteristics in relation to the maturational level of skiers. In order to reduce RAE in junior cross-country skiing, both coaches and policymakers in cross-country skiing should be aware of athletes' performance related to shortterm variables, which does not reflect the prospective potential of an athlete. Cross-country skiing may risk losing 'late bloomers' when inappropriate selection models and competition formats are used (duration time, track profiles) in junior groups. 


\section{Acknowledgements}

The authors of this study received no funding for this investigation and have no conflicts of interest.

\section{References}

Baker, J., Janning, C., Wong, H., Cobley, S., Schorer, J. (2014). Variations in relative age effects in individual sports: Skiing, figure skating and gymnastics. European Journal of Sport Science, 14(sup1), 183-190.

Barnsley, R. H., Thompson, A. H., Barnsley, P. E. (1985). Hockey success and birthdate: the relative age effect. CAHPER Journal, 51(8), 23-28.

Baxter-Jones, A. (1994). Born too late to win? Nature, 370, 186.

Bjerke, Ø., Pedersen, A. V., Aune, T. K., Lorås, H. (2017). An inverse relative age effect in male alpine skiers at the absolute top level. Frontiers in psychology, 8, 1210.

Cobley, S., Baker, J., Wattie, N., McKenna, J. (2009). Annual Age-Grouping and Athlete Development. Sports Medicine, 39(3), 235-256.

Cohen, J. (1978). Statistical power analysis for the behavioral sciences(Revised edition. ed.).

Dalen, T., Ingvaldsen, R. P., Roaas, T. V., Pedersen, Arve V., Steen, I., Aune, T. K. (2017). The impact of physical growth and relative age effect on assessment in physical education. European Journal of Sport Science, 17(4), 482-487.

de la Rubia, A., Lorenzo-Calvo, J., Lorenzo, A. (2020). Does the relative age effect influence short-term performance and sport career in team sports? A qualitative systematic review. Frontiers in Psychology, 11.

Delorme, N., Boiche, J., Raspaud, M. (2010). Relative age and dropout in French male soccer. Journal of Sports Sciences, 28(7), 717-722.

Delorme, N., Raspaud, M. (2009). The relative age effect in young French basketball players: a study on the whole population. Scandinavian Journal of Medicine \& Science in Sports, 19(2), 235-242.

Dudink, A. (1994). Birth date and sporting success. Nature, 368, 592.

Edgar, S., O'Donoghue, P. (2005). Season of birth distribution of elite tennis players. Journal of Sports Sciences, 23(10), 1013-1020.

Fonseca, F. S., Figueiredo, L. S., Gantois, P., de Lima-Junior, D., Fortes, L. S. (2019). Relative age effect is modulated by playing position but is not related to competitive success in elite under-19 handball athletes. Sports, 7(4), 91.

Gibbs, B. G., Shafer, K., Dufur, M. J. (2015). Why infer? The use and misuse of population data in sport research. International review for the sociology of sport, 50(1), 115-121.

Gulbin, J., Weissensteiner, J., Oldenziel, K., Gagné, F. (2013). Patterns of performance development in elite athletes. European Journal of Sport Science, 13(6), 605-614.

Hancock, D. J., Adler, A. L., Côté, J. (2013). A proposed theoretical model to explain relative age effects in sport. European Journal of Sport Science, 13(6), 630-637.

Hancock, D. J., Starkes, J. L., Ste-Marie, D. M. (2015). The relative age effect in female gymnastics: A flip-flop phenomenon. International Journal of Sport Psychology, 46(6), 714-725.

Helsen, W. F., Starkes, J. L., Van Winckel, J. (1998). The influence of relative age on success and dropout in male soccer players. American Journal of Human Biology, 10(6), 791-798.

Hirose, N. (2009). Relationships among birth-month distribution, skeletal age and anthropometric characteristics in adolescent elite soccer players. Journal of Sports Sciences, 27(11), 1159-1166.

Jones, C., Visek, A. J., Barron, M. J., Hyman, M., Chandran, A. (2019). Association between relative age effect and organisational practices of American youth football. Journal of Sports Sciences, 37(10), 1146-1153.

Kearney, P. E., Hayes, P. R., Nevill, A. (2018). Faster, higher, stronger, older: relative age effects are most influential during the youngest age grade of track and field athletics in the United Kingdom. Journal of Sports Sciences, 36(20), 2282-2288.

MacDonald, D., Cheung, M., Côté, J., Abernethy, B. (2009). Place but not Date of Birth Influences the Development and Emergence of Athletic Talent in American Football. Journal of Applied Sport Psychology, 21(1), 80-90.

McCarthy, N., Collins, D. (2014). Initial identification \& selection bias versus the eventual confirmation of talent: evidence for the benefits of a rocky road? Journal of Sports Sciences, 32(17), 1604-1610.

(c) Editorial Committee of Journal of Human Kinetics 
Merton, R. K. (1968). The Matthew Effect in Science. The reward and communication systems of science are considered, 159(3810), 56-63.

Musch, J., Grondin, S. (2001). Unequal competition as an impediment to personal development: a review of the relative age effect in sport. Developmental Review, 21(2), 147-167.

Müller, L., Müller, E., Kornexl, E., Raschner, C. (2015). The Relationship Between Physical Motor Skills, Gender and Relative Age Effects in Young Austrian Alpine Ski Racers. International Journal of Sports Science \& Coaching, 10(1), 69-85.

Newell, K. M. (1986). Constraints on the Development of Coordination. In M. G. Wade and H. T. A. Whiting (Eds.), Motor Development in Children Aspects of Coordination and Control (pp. 341-360). Martinus Nijhoff Publishers.

Nykodým, J., Bozděch, M., Agricola, A., \& Zháněl, J. (2020). The Relative Age Effect at the Ice Hockey World Championships (IHWC) in the years 2015-2017. Journal of Human Kinetics, 75, 150.

Raschner, C., Müller, L., Hildebrandt, C. (2012). The role of a relative age effect in the first winter Youth Olympic Games in 2012. British Journal of Sports Medicine, 46(15), 1038-1043.

Romann, M., Rössler, R., Javet, M., Faude, O. (2018). Relative age effects in Swiss talent development - a nationwide analysis of all sports. Journal of Sports Sciences, 36(17), 2025-2031.

Rosenthal, R., Jacobson, L. (1968). Pygmalion in the classroom: Teacher expetationan pupil's intellectual development. New York: Holt, Rinehart \& Winston.

Sandbakk, Ø., Holmberg, H.-C. (2017). Physiological Capacity and Training Routines of Elite Cross-Country Skiers: Approaching the Upper Limits of Human Endurance. International Journal of Sports Physiology $\mathcal{E}$ Performance, 12(8), 1003-1011.

Schorer, J., Cobley, S., Büsch, D., Bräutigam, H., Baker, J. (2009). Influences of competition level, gender, player nationality, career stage and playing position on relative age effects. Scandinavian Journal of Medicine E Science in Sports, 19(5), 720-730.

Solli, G. S., Kocbach, J., Seeberg, T. M., Tjønnås, J., Rindal, O. M. H., Haugnes, P., Sandbakk, Ø. (2018). Sexbased differences in speed, sub-technique selection, and kinematic patterns during low- and highintensity training for classical cross-country skiing. Plos One, 13(11).

Steingröver, C., Wattie, N., Baker, J., Schorer, J. (2016). Does Relative Age Affect Career Length in North American Professional Sports? Sports Medicine - Open, 2(1), 18.

Thorpe, H., Wheaton, B. (2011). 'Generation X Games', Action Sports and the Olympic Movement: Understanding the Cultural Politics of Incorporation. Sociology, 45(5), 830-847.

Wattie, N., Schorer, J., Baker, J. (2015). The Relative Age Effect in Sport: A Developmental Systems Model. Sports Medicine, 45(1), 83-94.

Webdale, K., Baker, J., Schorer, J., Wattie, N. (2020). Solving sport's 'relative age' problem: a systematic review of proposed solutions. International Review of Sport \& Exercise Psychology, 13(1), 187-204.

Wheaton, B. (2004). Understanding Lifestyle Sport: Consumption, Identity and Difference (B. Wheaton Ed.): Routledge.

\section{Internet resources}

1. www.skiforbundet.no

2. www.fis-ski.com

3. https://en.wikipedia.org/wiki/Freeskiing

4. http://afpjudges.com/general-info/judging-philosophy

\section{Corresponding author:}

\section{Truls Roaas}

Nord University

Hogskolevegen, N-7600 Levanger, Norway

Phone: +47 74022529

E-mail: truls.v.roaas@nord.no 